\title{
Peroxiredoxin 1 suppresses apoptosis via regulation of the apoptosis signal-regulating kinase 1 signaling pathway in human oral leukoplakia
}

\author{
MIN ZHANG* , WENWEN NIU* , JIANFEI ZHANG, LIHUA GE, JING YANG, ZHENG SUN and XIAOFEI TANG \\ Beijing Institute of Dental Research, Beijing Key Laboratory, Beijing Stomatological Hospital and School of Stomatology, \\ Capital Medical University, Beijing 100050, P.R. China
}

Received August 20, 2014; Accepted April 30, 2015

DOI: $10.3892 / \mathrm{ol} .2015 .3424$

\begin{abstract}
Peroxiredoxin 1 (Prx1) has a significant role in several malignant types of tumor. However, the role of Prx1 in oral leukoplakia (OLK) has remained to be elucidated. OLK is a common precancerous lesion of the oral mucosa that has a very high malignant transformation rate. The aim of the present study was to investigate the roles of Prx1, and its association with apoptosis signal-regulating kinase 1 (ASK1) and p38 in OLK. A total of 20 OLK samples and 10 normal oral mucosa samples were obtained from patients at the Beijing Stomatological Hospital (Beijing, China). The messenger RNA (mRNA) and protein expression levels of Prx1, ASK1 and p38 were determined by polymerase chain reaction and western blot analysis, respectively. Flow cytometry was used to detect cell apoptosis. The interaction between Prx1 and ASK1 was examined in $\mathrm{H}_{2} \mathrm{O}_{2}$-treated DOK cells by glutathione-S-transferase pull-down assays and by co-immunoprecipitation in vitro. Compared with those of the normal oral mucosa, the mRNA levels of Prx1, ASK1 and $\mathrm{p} 38$ were elevated in OLK tissues $(\mathrm{P}<0.05)$. The protein expression levels of Prx1, phosphorylated-ASK1 (p-ASK1) and p-p38 were also significantly enhanced in OLK tissues compared with those of the normal mucosa $(\mathrm{P}<0.05)$. In Prx1-knockdown DOK cells, ASK1 and p38 were activated, leading to enhanced levels of apoptosis in response to $\mathrm{H}_{2} \mathrm{O}_{2}$. No clear interaction between Prx1 and ASK1 was detected in $\mathrm{H}_{2} \mathrm{O}_{2}$-treated DOK cells. Prx1 was suggested to be involved
\end{abstract}

Correspondence to: Professor Xiaofei Tang, Beijing Institute of Dental Research, Beijing Key Laboratory, Beijing Stomatological Hospital and School of Stomatology, Capital Medical University, 4 Tiantan Xili, Beijing 100050, P.R. China

E-mail: tangxf0401@sohu.com

*Contributed equally

Key words: oral leukoplakia, peroxiredoxin 1, apoptosis, apoptosis signal-regulating kinase 1, p38 in OLK pathogenesis by providing resistance against extracellular damages from oxidative stress via inhibition of the ASK1-induced apoptotic signaling pathway. Targeting Prx1 may provide a novel therapeutic strategy for the treatment of patients with OLK.

\section{Introduction}

Oral cancer accounts for $\sim 3 \%$ of all malignancies worldwide, with $\sim 500,000$ new cases diagnosed annually and a five-year survival rate of $\sim 50 \%$ (1). The development of oral cancer is usually preceded by the occurrence of precancerous lesions. Oral leukoplakia (OLK), also known as a common oral precancerous lesion, is a type of oral mucosal epithelial keratosis abnormality. The global prevalence of OLK is $\sim 1 \%$ for all ages, with an increasing prevalence in adults, and the malignant transformation rate of OLK is $2-3 \%$ (2). Leukoplakia usually occurs $\sim 5$ years prior to the development of oral cancer (3). At present, no specific treatment has been identified for OLK, thus preventing the development and progression of OLK is important as it may reduce the incidence of oral cancer. Previous studies have demonstrated that oxidative stress damage may be involved in the pathogenesis of OLK (4,5). Peroxiredoxin 1 (Prx1) is a major 2-Cys member of the peroxiredoxin family, which is abundant and ubiquitously distributed in tissues and is expressed at higher levels in numerous types of malignant tumor, including oral squamous cell carcinoma (6-8). The primary biochemical function of Prx1 appears to be as a peroxide-detoxifying enzyme scavenging reactive oxygen species (ROS), and studies have identified its functional switching from a peroxidase enzyme to a molecular chaperone, which regulates cell proliferation, differentiation and apoptosis under stress conditions $(9,10)$. Apoptosis signal-regulating kinase 1 (ASK1) is well known as a proapoptotic, stress-acivated signaling molecule, which participates in the c-Jun N-terminal kinase (JNK) and p38-mitogen activated protein kinase (MAPK) signaling cascades (11). It has been reported that Prx1 interacts with ASK1 via the thioredoxin-binding domain of ASK1, and that this action is highly inducible by $\mathrm{H}_{2} \mathrm{O}_{2}$ (12). However, to the best of our knowledge, no information is currently available regarding the role of Prx1 in OLK. In the present study, for 
the first time, to the best of our knowledge, the role of Prx1 in ASK1-induced apoptosis by oxidative stress in OLK was investigated, in order to provide valuable clues for the prevention and treatment of OLK.

\section{Materials and methods}

Patients and specimens. A total of 20 OLK patients with clinical and pathological diagnosis of OLK, with epithelial mild or mild-moderate dysplasia, at the Capital Medical University School of Stomatology (Beijing, China) were randomly selected for use in the present study. The OLK tissues of these patients were taken via biopsy, and 10 samples of normal oral mucosa were obtained from maxillofacial plastic surgery procedures for use as negative controls. Amongst the 20 OLK cases, 12 were female and 8 were male, aged 45-84 years (mean age, 64 years), including 13 cases of buccal mucosa, 1 case of lip mucosa and 6 cases of tongue mucosa. The present study was approved by the Human Research Ethics Committee of Capital Medical University School of Stomatology, and all patients signed an informed consent forms.

Reverse transcription-quantitative polymerase chain reaction (RT-qPCR). For quantification of messenger RNA (mRNA) expression, total RNA was extracted from human OLK and control tissues using TRIzol (Invitrogen Life Technologies, Carlsbad, CA, USA) according to the manufacturer's instructions. Complementary DNA (cDNA) was synthesized by reverse transcribing $2 \mu \mathrm{g}$ RNA with the High-Capacity cDNA Reverse Transcription kit (Applied Biosystems, Foster City, CA, USA). Aliquots of cDNA ( $1 \mu \mathrm{l})$ were used as templates and SYBR Green Dye reagent (Applied Biosystems, Foster City, CA, USA) was used to quantify the products formed during the RT-qPCR reaction. For data analysis, the $2^{-\Delta \Delta \mathrm{Ct}}$ method was used, and the raw data was normalized to the housekeeping gene GAPDH (13). The experiment was performed in triplicate. The sequences of primers were: GAPDH-forward (F), 5'-aggtcggtgtgaacggatttg-3' and reverse (R), 5'-tgtagaccatgtagttgaggtca-3'; Prx1-F, 5'-gggtattcttcggcagatca-3' and Prx1-R, 5'-tcccatgtttgtcagtgaa-3'; ASK1-F, 5'-aagtcccaacccatagaaattcct-3' and ASK1-R, 5'-agccagtcggtaagttcagaatctt-3'; p38-F, 5'-gagctgaagattctggattttgg-3' and p38-R, 5'-tagccacgtagccggtcatt-3'.

Cell culture. Human oral precancerous cell line DOK (presented by Professor Chen Xiaoxin, Cancer Research Program, Julius L. Chambers Biomedical/Biotechnology Research Institute, North Carolina Central University, Durham, NC, USA) were maintained in Dulbecco's modified Eagle's medium-nutrient mixture F-12, supplemented with $15 \%$ (v/v) fetal bovine serum (FBS; Gibco Life Technologies, Carlsbad, CA, USA) containing $100 \mathrm{U} / \mathrm{ml}$ penicillin and $100 \mu \mathrm{g} / \mathrm{ml}$ streptomycin, in a $5 \% \mathrm{CO}_{2}$ atmosphere at $37^{\circ} \mathrm{C}$.

Plasmids and cell transfection. The pEZ-M02-ASK1 and pEZ-M02-Prx1 plasmids were obtained from GeneCopoeia, Inc. (Rockville, MD, USA), while Prx1 short hairpin RNA (shRNA) plasmid and control shRNA Plasmid-A were obtained from Santa Cruz Biotechnology, Inc. (Dallas, TX, USA). DOK cells were plated in six-well plates at $1 \times 10^{6}$ cells/well, then transfected with $2 \mu \mathrm{g}$ plasmids using Lipofectamine ${ }^{\circledR} 2000$ (Invitrogen Life Technologies) according to the manufacturer's instructions.

Flow cytometry for cell apoptosis detection. Following transfection of Prx1 shRNA plasmid for $48 \mathrm{~h}$, the cells were stimulated with $5 \mathrm{mM} \mathrm{H}_{2} \mathrm{O}_{2}$ for various time-periods (15, 30 and $45 \mathrm{~min}$ ). Apoptotic cell death was measured by flow cytometry (FACSCalibur; BD Biosciences, Franklin Lakes, NJ, USA) with Annexin V/fluorescein isothiocyanate and propidium iodide staining (R\&D Systems, Inc., Minneapolis, MN, USA).

Western blot analysis. Cells and tissues were rinsed three times with ice-cold phosphate-buffered saline (PBS; Hyclone, Logan, UT, USA) and lysed in immunoprecipitation assay buffer [50 mM Tris-Cl (pH 7.4), 1\% NP40, 150 Mm NaCl, $1 \mathrm{mM}$ EDTA, $1 \mathrm{M}$ phenylmethylsulfonyl fluoride, $10 \mu \mathrm{g}$ each of aprotinin and leupeptin, and $1 \mathrm{mM} \mathrm{Na}_{3} \mathrm{VO}_{4}$ ] (Invitrogen Life Technologies), to which the protease inhibitor mix Complete $^{\mathrm{TM}}$ (Roche Diagnostics, Basel, Switzerland) was added. Following centrifugation at $12,000 \times \mathrm{g}$ for $30 \mathrm{~min}$, the supernatant was collected, and the protein concentration was determined using the Lowry method (14). Equal quantities of protein were separated on $12 \%$ SDS-PAGE gels and blotted onto nitrocellulose membranes (Pierce Biotechnology, Inc., Appleton, WI, USA). The blots were subsequently incubated with rabbit polyclonal anti-human $\operatorname{Prx} 1$ antibody $(1: 1,000$; cat. no. ab41906; Abcam, Cambridge, UK), rabbit polyclonal anti-human ASK1 (1:1,000; cat. no. 3762s; Cell Signaling Technology, Inc., Danvers, MA, USA), rabbit polyclonal anti-human p-ASK1 (1:1,000; cat. no. 3765s; Cell Signaling Technology, Inc.), rabbit monoclonal anti-human p38 (1:1,000; cat. no. ab7952; Abcam) and rabbit monoclonal anti-p-human p38 (1:1,000; cat. no. ab178867; Abcam) at $4^{\circ} \mathrm{C}$ overnight. Rabbit polyclonal anti-human $\beta$-actin antibody (1:1,000; cat. no. A2066; Sigma-Aldrich, St. Louis, MO, USA) was used as a loading control. Immunoreactive bands were detected by $1-\mathrm{h}$ incubation at room temperature with goat anti-rabbit (cat. no. ab136636) and goat anti-mouse (cat. no. ab979023) horseradish peroxidase (HRP)-conjugated secondary antibodies (1:1,000; Abcam) and enhanced chemiluminescence reagents (GE Healthcare Life Sciences, Chalfont, UK). Each experiment was repeated a minimum of three times.

Co-immunoprecipitation assay. To examine the association between Prx1 and ASK1, a co-immunoprecipitation assay was used to detect the interaction between Prx1 with ASK1 in vitro. Cell extract from $\mathrm{H}_{2} \mathrm{O}_{2}$-treated DOK cells was collected following centrifugation at 14,000 $\mathrm{x} g$ for $15 \mathrm{~min}$ at $4^{\circ} \mathrm{C}$ and boiled for 5 min with $2 \mathrm{X}$ loading buffer for examination by $4-12 \%$ SDS-PAGE. Whole cell extracts were incubated with the rabbit monoclonal anti-human Prx1 (1:1,000; cat. no. ab109506; Abcam) or rabbit polyclonal anti-human $\beta$-actin (1:1,000; cat. no. A2066; Sigma-Aldrich) antibodies at $4{ }^{\circ} \mathrm{C}$ overnight and incubated with the HRP-conjugated secondary antibodies for $30 \mathrm{~min}$ at room temperature. For protein precipitation, protein A-Agarose beads (Invitrogen Life Technologies) in extract buffer were 


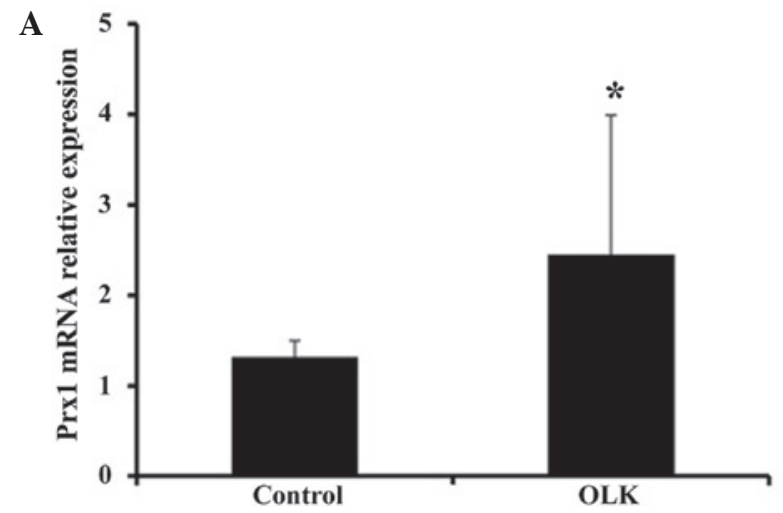

B

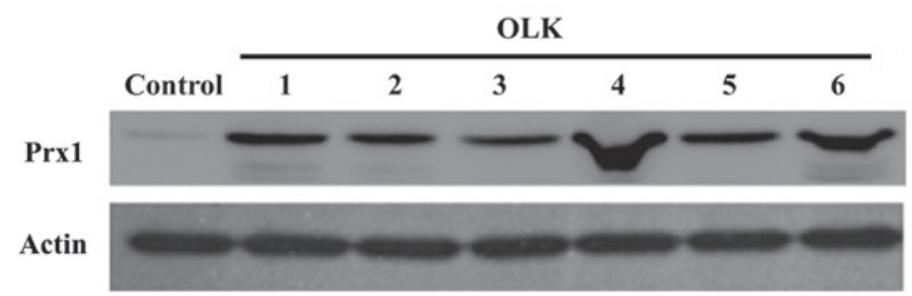

Figure 1. Prx1 mRNA and protein expression is enhanced in OLK tissues. (A) Total RNA was isolated and quantified by reverse transcription-quantitative polymerase chain reaction. Fold change values were normalized to GADPH levels. Values are expressed as the mean \pm standard deviation of triplicate experiments; ${ }^{*} \mathrm{P}<0.05$ vs. control. (B) Equal aliquots of protein were subjected to western blot analysis with antibodies specific to Prx 1 and $\beta$-actin. $\beta$-actin was used as internal loading control. The control and 6 samples were chosen at random and the experiments were repeated in triplicate. Prx1, peroxiredoxin 1; mRNA, messenger RNA; OLK, oral leukoplakia.

A

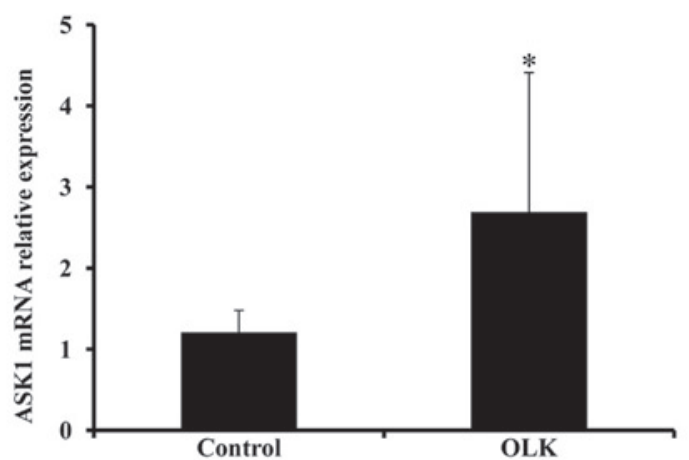

C

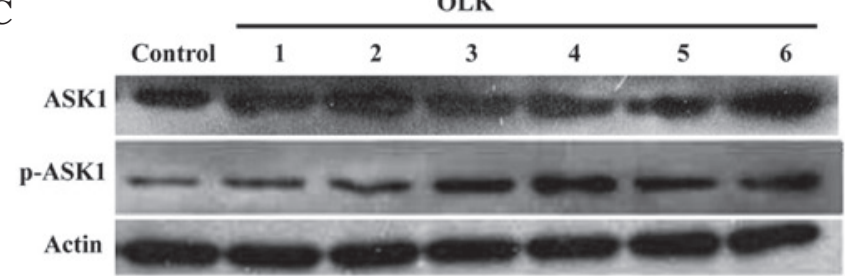

B

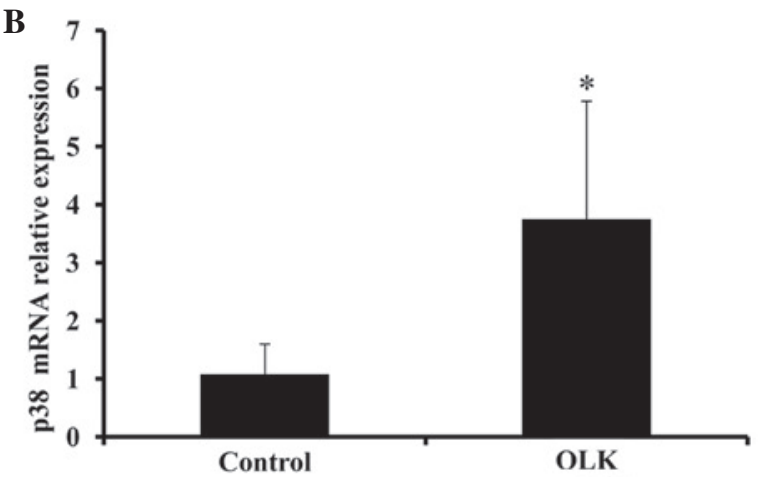

D

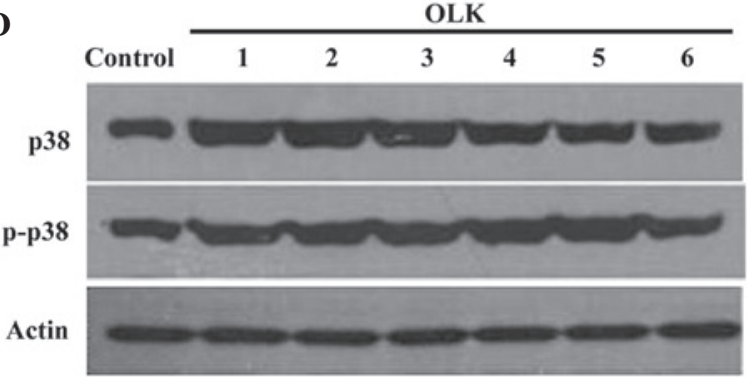

Figure 2. ASK1 and p38 mRNA and protein expression is enhanced in OLK tissues. Total (A) ASK1 and (B) p38 RNA was isolated and quantified by reverse transcription-quantitative polymerase chain reaction. Fold change values were normalized to GADPH levels. Values are expressed as the mean \pm standard deviation of triplicate experiments; " $\mathrm{P}<0.05$ vs. control. Equal aliquots of (C) ASK1 and (D) p38 protein were subjected to western blot analysis with anti-ASK1, anti-p-ASK1, anti-p38, anti-p-p38 and anti- $\beta$-actin antibodies. $\beta$-actin was used as internal loading control. The control and 6 samples were chosen at random and the experiments were repeated in triplicate. ASK1, apoptosis signal-regulating kinase 1; mRNA, messenger RNA; OLK, oral leukoplakia; p, phosphorylated.

added prior to incubation with gentle mixing for $16 \mathrm{~h}$ at $4{ }^{\circ} \mathrm{C}$. Subsequently, the beads were pelleted by centrifugation at $14,000 \mathrm{x} \mathrm{g}$ for $15 \mathrm{~min}$ at $4^{\circ} \mathrm{C}$ and washed three times with extract buffer. The protein was eluted from the beads at $100^{\circ} \mathrm{C}$ using $1 \%$ SDS-PAGE sample buffer supplemented with $50 \mathrm{mM}$ dithiothreitol and resolved with SDS-PAGE.

Glutathione-S-transferase (GST) pull-down assays. To further investigate whether Prx1 protein interacts with ASK1 protein directly in vitro, a GST pull-down assay kit (21516; Thermo Fisher Scientific, Waltham, MA, USA) was used. Cell lysates were prepared and centrifuged at $15,000 \mathrm{x} \mathrm{g}$ for $15 \mathrm{~min}$ and the supernatants were collected. Histidine (His)-Prx1 (Prx1 protein with a 6-His tag, prepared in our laboratory) were incubated with GST or GST-fused ASK1 conjugated to sepharose beads in reaction buffer $(50 \mathrm{mM}$ Tris- $\mathrm{HCl}, \mathrm{pH} 7.5,150 \mathrm{mM} \mathrm{NaCl}, 10 \%$ glycerol, $1.5 \mathrm{mM}$ $\mathrm{MgCl}_{2}, 5 \mathrm{mM} \mathrm{NaF}, 1 \%$ Triton X-100 and protease inhibitor mixture Complete; Roche Diagnostics) at $4^{\circ} \mathrm{C}$ for $12 \mathrm{~h}$. Following centrifugation at $500 \mathrm{x} \mathrm{g}$ for $5 \mathrm{~min}$ at $4^{\circ} \mathrm{C}$, the proteins bound to sepharose beads were washed with ice-cold PBS, mixed with $2 X$ SDS sample buffer and eluted by the addition of $10 \mu \mathrm{l}$ glutathione elution buffer (G-Biosciences, St. Louis, MO, USA). The sepharose beads were suspended 
A

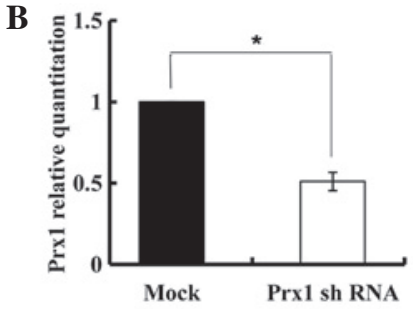

C

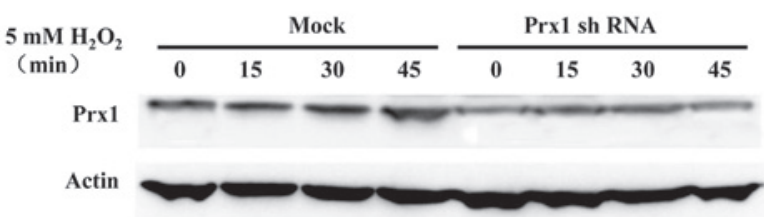
Prx1 sh RNA

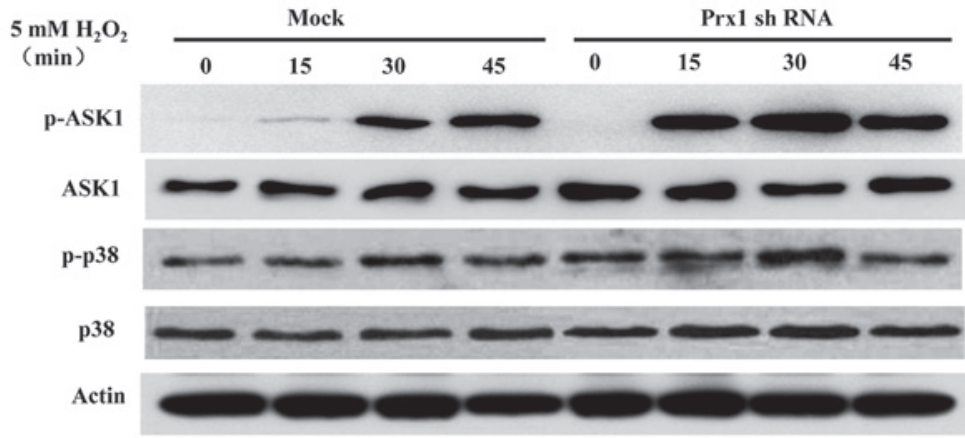

Figure 3. Expression of Prx1 and its regulation of the ASK1-mediated signaling pathway for the activation of p38. DOK cells were transfected with control and Prx1 shRNA vector. Following transfection for $48 \mathrm{~h}$, cells were treated with $5 \mathrm{mM} \mathrm{H}_{2} \mathrm{O}_{2}$ for the indicated times, and cell lysates were subjected to immunoblot analysis with anti-Prx1, anti-p-Thr845 ASK1, anti-ASK1, anti-p-p38, anti-p38 or anti- $\beta$-actin antibodies. (A) Expression of $\operatorname{Prx}_{1}$ in $\mathrm{H}_{2} \mathrm{O}_{2}$-treated DOK cells, with or without Prx1 shRNA transfection. (B) Prx1 expression was suppressed by shRNA transfection, ${ }^{*} \mathrm{P}=0.046$. (C) The expression of ASK1, p-ASK1, p38 and p-p38 in DOK cells with or without Prx1 shRNA transfection. Prx1, peroxiredoxin 1; mRNA, messenger RNA; OLK, oral leukoplakia; ASK1, apoptosis signal-regulating kinase 1; p, phosphorylated; shRNA, short hairpin RNA.

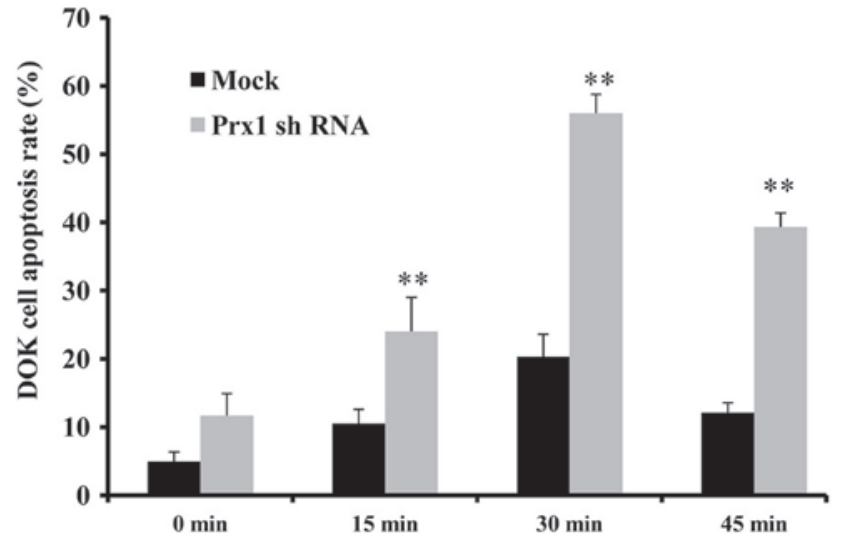

Figure 4. $\mathrm{H}_{2} \mathrm{O}_{2}$-induced apoptosis is enhanced in Prx1 knockdown cells Following transfection for $48 \mathrm{~h}$, cultured cells were treated with $5 \mathrm{mM}$ $\mathrm{H}_{2} \mathrm{O}_{2}$ for $0,15,30$ and $45 \mathrm{~min}$. Apoptotic cell death was measured by flow cytometry. Data are represented as the mean of triplicate independent experiments \pm standard error of the mean. ${ }^{* *} \mathrm{P}<0.01$ vs. mock. Prx1, peroxiredoxin 1 ; shRNA, short hairpin RNA.

and incubated at room temperature for $5 \mathrm{~min}$. They were then centrifuged for at $500 \mathrm{x} \mathrm{g}$ for $5 \mathrm{~min}$ at $4^{\circ} \mathrm{C}$ to sediment the sepharose beads, and the supernatants were transferred to fresh tubes for SDS-PAGE. Western blot analysis was then performed to examine Prx1 binding to ASK1 using rabbit monoclonal anti-human Prx1 antibody $(1: 1,000$; cat. no. ab109506; Abcam).

Statistical analysis. Data were expressed as the mean \pm standard deviation. Comparisons were performed by two independent Student's t-test (independent 2-sample t-test) using SPSS 17.0 (SPSS, Inc., Chicago, IL, USA). P $<0.05$ was considered to indicate a statistically significant difference.

\section{Results}

Prxl expression is enhanced in OLK tissues. Prx 1 mRNA expression was significantly higher in 20 OLK tissues than that of normal oral mucosa samples, with approximately 2 -fold greater expression than that of the normal group ( $\mathrm{P}=0.047$; Fig. 1A). Closely correlated with the mRNA levels, the protein expression of Prx 1 was also enhanced in 20 cases of OLK, compared with that of the normal group, and the mean relative Prx1 content was 1.11 in the OLK group (Fig. 1B).

Expression levels of ASK1, p-ASK1, p38 and p-p38 are increased in OLK tissues. The mRNA expression levels of ASK1 and p38 were both significantly higher in OLK tissues than that in the normal oral mucosa, with $\sim 3-(\mathrm{P}=0.024)$ (Fig. 2A) and 4-fold ( $\mathrm{P}=0.022)$ (Fig. 2B) increase compared with the normal group, respectively. The protein expression of ASK1 and p-ASK1 were slightly increased in the OLK tissues, and the mean relative content of ASK1 and p-ASK1 was 1.06 and 1.11, respectively, in the OLK group (Fig. 2C). The protein expression of p38 and p-p38 in OLK group were also both increased slightly, and the mean relative $\mathrm{p} 38$ and p-p38 content was 1.09 and 1.11, respectively, in the OLK group (Fig. 2D) The results of the western blot were not statistically analyzed.

Expression of Prxl, ASK1 and p38 in DOK cells treated with $\mathrm{H}_{2} \mathrm{O}_{2}$. Western blot analysis with anti-Prx1 antibody was performed in DOK cells treated with $5 \mathrm{mM} \mathrm{H}_{2} \mathrm{O}_{2}$ for the indicated time-periods (Fig. 3A). The expression of Prx1 was not markedly altered until $45 \mathrm{~min}$. To investigate whether endogenous Prx1 affected ASK1 expression and ASK1-induced apoptosis, the suppression of endogenous 
A
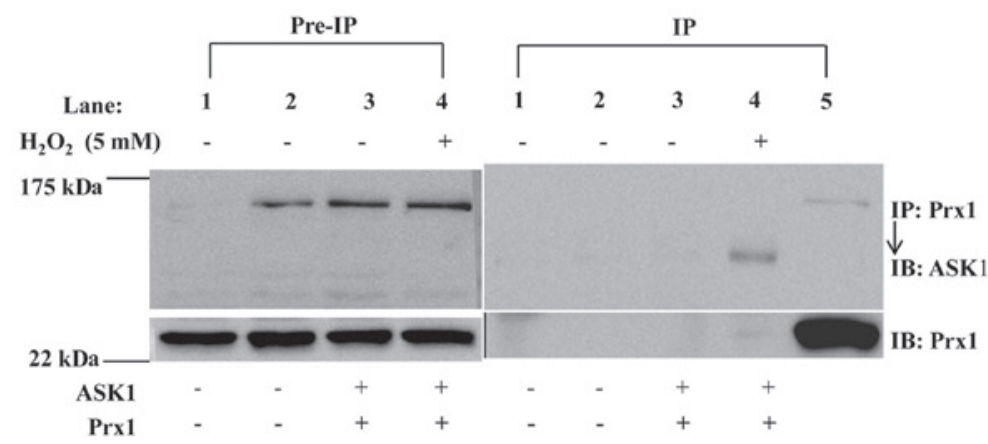

B

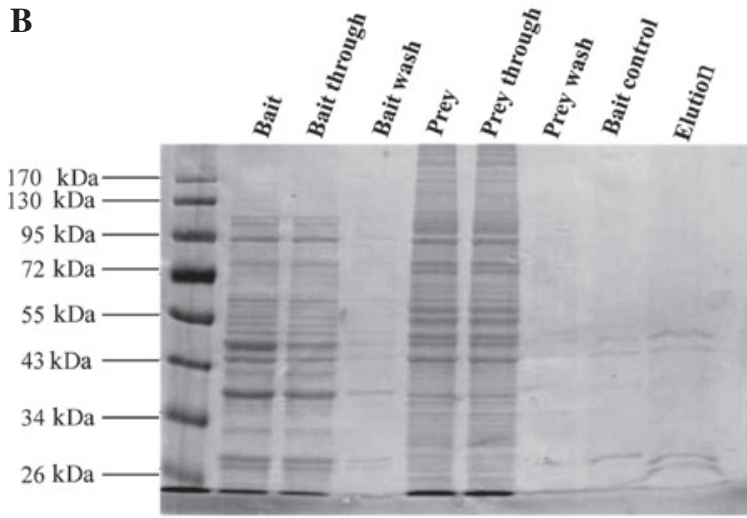

Figure 5. No interaction was identified between Prx1 and ASK1. (A) DOK cells were transfected for $48 \mathrm{~h}$ with plasmids encoding Prx1 and ASK1, respectively. Cells were stimulated with $5 \mathrm{mM} \mathrm{H} \mathrm{O}_{2}$ for $30 \mathrm{~min}$. Cell lysates were incubated with rabbit monoclonal antibodies against Prxl, precipitated by Protein A/G beads and detected by western blots using rabbit polyclonal antibodies against ASK1. Pre-immune IgG was used as a negative control. Lane 1, blank cell control; lane 2, transfection control; lane 3, Cells transfected with 2 vectors; lane 4, cells transfected with 2 vectors and treated with $\mathrm{H}_{2} \mathrm{O}_{2}$; lane 5, positive control. (B) Direct interaction between Prx1 and ASK1 proteins in vitro was investigated by glutathione S-transferase pull-down assay. Prx1, peroxiredoxin 1; ASK1, apoptosis signal-regulating kinase 1; IP, immunoprecipitation; IB, immunoblotting; IgG, goat anti-rabbit HRP-conjugated IgG.

Prx1 expression was induced by transfecting Prx1 shRNAs into DOK cells (Fig. 3B). The expression of phosphorylated ASK1 and p38 were subsequently evaluated by immunoblotting assay. As shown in Fig. 3C, upon stimulation with $\mathrm{H}_{2} \mathrm{O}_{2}$, the levels of p-ASK1 rose rapidly and strongly from 15 min. Following Prx1 knockdown, higher levels of p-ASK1 and p-p38 were observed, compared with that of control (mock-transfected) DOK cells. In Prx1-knockdown DOK cells, the expression of phosphorylated ASK1 and p38 gradually increased in a time-dependent manner, and then decreased at $45 \mathrm{~min}$.

Prxl suppresses apoptosis in DOK cells treated with $\mathrm{H}_{2} \mathrm{O}_{2}$. Subsequently, the functional roles of $\operatorname{Prx} 1$ in $\mathrm{H}_{2} \mathrm{O}_{2}$-induced apoptosis were examined. DOK cells were transiently transfected with Prx1 shRNA. Following transfection for $48 \mathrm{~h}$, the cells were treated with $5 \mathrm{mM} \mathrm{H}_{2} \mathrm{O}_{2}(0,15,30$ and $45 \mathrm{~min})$ and apoptotic cell death was measured by flow cytometry. Notably, $\mathrm{H}_{2} \mathrm{O}_{2}$-induced apoptosis was significantly enhanced in DOK cells transfected with Prx1 shRNA compared with that of the mock-transfected cells (Fig. 4), particularly following treatment with $5 \mathrm{mM} \mathrm{H}_{2} \mathrm{O}_{2}$ for $30 \mathrm{~min}(56.0 \pm 2.8 \mathrm{vs}$. $20.3 \pm 3.3 \%$, respectively; $\mathrm{P}=0.008$ ).

No interaction is detected between Prxl and ASK1 in DOK cells treated with $\mathrm{H}_{2} \mathrm{O}_{2}$. To investigate the association between $\operatorname{Prx} 1$ and ASK1, whether Prx1 interacts with ASK1 in vitro was examined (Fig. 5A). DOK cells were transiently transfected with expression plasmids encoding ASK1 and Prx1. Following co-transfection for $48 \mathrm{~h}$, the cells were stimulated with $5 \mathrm{mM}$ of $\mathrm{H}_{2} \mathrm{O}_{2}$ for $30 \mathrm{~min}$, extracted and immunoprecipitated with anti-Prx1 antibody. No ASK1 was detected in immunoprecipitates of endogenous Prx 1 under these conditions.

Whether Prx1 directly interacts with ASK1 in vitro was further examined by GST pull-down assay. A GST pull-down assay kit was used, and the results indicated that ASK1 did not markedly bind to Prx1 (Fig. 5B). These results suggested that Prx1 and ASK1 were not able to directly interact in vitro under these conditions.

\section{Discussion}

Studies have shown that the pathogenesis of tumors is closely associated with oxidative stress. The levels of ROS reflect the intracellular redox state, and in turn, high levels of ROS induce DNA damage or cell death $(15,16)$. Previous studies by our group revealed that the development of OLK was also associated with oxidative stress (17). Peroxiredoxins are a family of peroxidases, which catalyze the removal of $\mathrm{H}_{2} \mathrm{O}_{2}$ and other hydroperoxides. Prx1 is a major member of the peroxiredoxin family, and is localized to the cytoplasm, functioning to protect cells from excessive ROS damage (18). The expression of Prx1 is increased in multiple malignant tumors, including oral cancer $(6,19)$. It was previously reported that the overexpression of Prx1 was able to enhance the clonogenic survival of irradiated cells and attenuate ionizing radiation-induced JNK activation and apoptosis (10). Prx1 inhibits cell apoptosis by regulating the activation of the p38MAPK signaling pathway in cisplatin-induced oxidative stress (7). A previous study by our group demonstrated that the expression of Prx1 was significantly higher in human OLK tissues than that in the normal oral mucosa (17). Prx1 expression is closely associated with the apoptosis in OLK tissues (20). In the present study, compared with the normal oral mucosa, the mRNA and protein expression levels of Prx1, ASK1 and p38 were increased, and p-ASK1 and p-p38 were overexpressed in OLK tissues $(\mathrm{P}<0.05)$. These results suggest that Prx1 may be involved in the development of OLK, and is associated with oxidative stress-induced apoptosis, however, the specific molecular mechanism remains elusive.

ASK1, a kinase functioning upstream of the JNK activating signaling cascade, performs distinct biological functions in cell differentiation and regulation of apoptotic signaling in response to external stimulation $(21,22)$. The $\mathrm{p} 38$ protein has a wide range of biological functions, including roles in cell proliferation, differentiation, apoptosis, migration and invasion. As a regulator of cell apoptosis, p38 exerts dual functions in regulating cell survival or apoptosis depending on the diversity of cell types and external stimuli (23). Certain studies have suggested that p38 is highly expressed and has significant roles 
in prostate, breast, bladder and lung cancer, as well as follicular lymphoma and leukemia (24). When cells are subjected to oxidative stress, inflammation and other external stimuli, ASK1 is phosphorylated and subsequently activates the JNK and p38 downstream signaling pathways, $\mathrm{p}-\mathrm{JNK}$ and $\mathrm{p}$-p38, promoting cell apoptosis $(25,26)$. In thyroid cancer cells exposed to Prx1 inhibitor MG132, the expression levels of ASK1 and p38 were increased (27). A recent report demonstrated that, in human malignant breast epithelial cells, silencing of the Prx1 gene and treatment with various concentrations of $\mathrm{H}_{2} \mathrm{O}_{2}$, significantly increased p-p38 protein expression (9). The expression of Prx1 in dopaminergic neuronal cells inhibited 6-hydroxydopamine-induced apoptotic death by reducing p38/caspase-3 activation (28).

In order to investigate whether endogenous Prx1 affected ASK1-mediated apoptosis induced by oxidative stress in OLK, the effects of Prx1-knockdown on the activation of ASK1-mediated signaling by $\mathrm{H}_{2} \mathrm{O}_{2}$ in DOK cells was evaluated. Following stimulation of DOK cells with $\mathrm{H}_{2} \mathrm{O}_{2}$, the levels of p-ASK1 were rapidly and notably enhanced at $15 \mathrm{~min}$, while p-ASK 1 and p-p38 gradually increased in a time-dependent manner. Interestingly, the levels were subsequently reduced at 45 min. Following Prx1 knockdown, higher levels of p-ASK1 and p-p38 were observed, compared with that in the control (mock-transfected) DOK cells. The expression of p-ASK1 and p-p38 were gradually increased in a time-dependent manner. These results suggested that endogenous Prx1 may be a negative regulator for ASK1-mediated signaling in the activation of the 338 pathway in response to $\mathrm{H}_{2} \mathrm{O}_{2}$. In addition, $\mathrm{H}_{2} \mathrm{O}_{2}$-induced apoptosis was significantly enhanced in Prx1-knockdown DOK cells compared with that in mock-transfected cells, which suggest that Prx1 may function as an endogenous antagonist of ASK1-mediated apoptosis induced by $\mathrm{H}_{2} \mathrm{O}_{2}$, and $\mathrm{Prx} 1$ may exert a protective effect against ASK1-induced apoptosis mediated by oxidative stress. However, the specific mechanisms underlying the effects of Prx1, ASK1 and p38 in the development of OLK require further study.

The interaction of Prx1 with ASK1 in DOK cells was examined, and no direct interaction between ASK1 and Prx1 was observed in co-immunoprecipitation or GST pull-down assays, which differed to the results of a previous group, where interactions were identified between Prx 1 and ASK1 in response to $\mathrm{H}_{2} \mathrm{O}_{2}$ in HEK293 cells (12). The results indicated that Prx1 may interact with ASK1 indirectly, or that the formation of an interaction between ASK1 and Prx1 occurred in a transient manner or at a lower affinity that was undetectable in the specific conditions of the present study (29).

In conclusion, the present results indicated that Prx1 is involved in OLK pathogenesis. Prx1 may participate in providing resistance against extracellular damage from oxidative stress via inhibition of the ASK1-induced apoptotic signaling pathway. Therefore, targeting Prx1 may offer a novel strategy for the treatment of patients with OLK.

\section{Acknowledgements}

The present study was funded by the National Natural Science Foundation (no. 81070836), the Beijing Natural Science
Foundation (no. 7102065), the National Natural Science Foundation of China (no. 81470752) and the Beijing Municipal Administration of Hospital Key Medical Development Project (no. ZYLX201407).

\section{References}

1. Messadi DV: Diagnostic aids for detection of oral precancerous conditions. Int J Oral Sci 5: 59-65, 2013.

2. van der Waal I: Oral potentially malignant disorders: Is malignant transformation predictable and preventable? Med Oral Patol Oral Cir Bucal 19: e386-e390, 2014.

3. Neville BW and Day TA: Oral cancer and precancerous lesions. CA Cancer J Clin 52: 195-215, 2002.

4. Nayyar AS and Khan M: In search of malignant transformation: A pilot study. J Cancer Res Ther 8: 277-281, 2012.

5. Metgud R and Bajaj S: Evaluation of salivary and serum lipid peroxidation, and glutathione in oral leukoplakia and oral squamous cell carcinoma. J Oral Sci 56: 135-142, 2014.

6. Yanagawa T, Iwasa S, Ishii T, Tabuchi K, Yusa H, Onizawa K, Omura K, Harada H, Suzuki H and Yoshida H: Peroxiredoxin I expression in oral cancer: A potential new tumor marker. Cancer Lett 156: 27-35, 2000.

7. Cha MK, Suh KH and Kim IH: Overexpression of peroxiredoxin I and thioredoxin1 in human breast carcinoma. J Exp Clin Cancer Res 28: 93, 2009.

8. Zhao YH, Zhang M, Yan F, Casto BC and Tang XF: Nicotine-induced upregulation of antioxidant protein Prx 1 in oral squamous cell carcinoma. Chin Sci Bull 58: 1912-1918, 2013.

9. Turner-Ivey B, Manevich Y, Schulte J, Kistner-Griffin E, Jezierska-Drutel A, Liu Y and Neumann CA: Role for Prdx1 as a specific sensor in redox-regulated senescence in breast cancer. Oncogene 32: 5302-5314, 2013.

10. Kim YJ, Lee WS, Ip C, Chae HZ, Park EM and Park YM: Prx1 suppresses radiation-induced c-Jun NH2-terminal kinase signaling in lung cancer cells through interaction with the glutathione S-transferase $\mathrm{Pi} / \mathrm{c}$-Jun NH2-terminal kinase complex. Cancer Res 66: 7136-7142, 2006.

11. Turjanski AG, Vaqué JP and Gutkind JS: MAP kinases and the control of nuclear events. Oncogene 26: 3240-3253, 2007.

12. Kim SY, Kim TJ and Lee KY: A novel function of peroxiredoxin 1 (Prx-1) in apoptosis signal-regulating kinase 1 (ASK1)-mediated signaling pathway. FEBS Lett 582: 1913-1918, 2008.

13. Wu Z, Sheng H, Chen Y, Tang J, Liu Y, Chen Q, Lu L and Jin W: Copy number variation of the Lipoprotein(a) (LPA) gene is associated with coronary artery disease in a southern Han Chinese population. Int J Clin Exp Med 7: 3669-3677, 2014.

14. Lowry OH, Rosebrough NJ, Farr AL and Randall RJ: Protein measurement with the Folin phenol reagent. J Biol Chem 193: 265-275, 1951.

15. Memon AA, Chang JW, Oh BR and Yoo YJ: Identification of differentially expressed proteins during human urinary bladder cancer progression. Cancer Detect Prev 29: 249-255, 2005.

16. Xie Q, Zhou Y, Lan G, Yang L, Zheng W, Liang Y and Chen T: Sensitization of cancer cells to radiation by selenadiazole derivatives by regulation of ROS-mediated DNA damage and ERK and AKT pathways. Biochem Biophys Res Commun 449: 88-93, 2014.

17. Ge LH, Hou M, Yang J, Chen T and Tang XF: Prx1 overexpression in human OLK. Beijing J Stomatology 20: 135-137, 2012.

18. Chatterjee S, Feinstein SI, Dodia C, Sorokina E, Lien YC, Nguyen S, Debolt K, Speicher D and Fisher AB: Peroxiredoxin 6 phosphorylation and subsequent phospholipase A2 activity are required for agonist-mediated activation of NADPH oxidase in mouse pulmonary microvascular endothelium and alveolar macrophages. J Biol Chem 286: 11696-11706, 2011.

19. Tang XF, Zhang XY and Zhang M: The differences expression of oxidative stress-related genes in oral cancer and precancerous cells. Beijing J Stomatology 16: 308-311, 2008.

20. Zhang JF, Tang XF, Ge LH, Yang J, Niu WW, Zhang M and Chen T: Role of apoptosis signal-regulating kinase 1 in the cell apoptosis in oral leukoplakia. Beijing J Stomatology 22: 65-69, 2014.

21. Nakagawa H, Hirata Y, Takeda K, Hayakawa Y, Sato T, Kinoshita H, Sakamoto K, Nakata W, Hikiba Y, Omata M, et al: Apoptosis signal-regulating kinase 1 inhibits hepatocarcinogenesis by controlling the tumor-suppressing function of stress-activated mitogen-activated protein kinase. Hepatology 54: 185-195, 2011. 
22. Hayakawa Y, Hirata Y, Nakagawa H, Sakamoto K, Hikiba Y, Kinoshita H, Nakata W, Takahashi R, Tateishi K, Tada M, et al: Apoptosis signal-regulating kinase 1 and cyclin D1 compose a positive feedback loop contributing to tumor growth in gastric cancer. Proc Natl Acad Sci USA 108: 780-785, 2011.

23. Koul HK, Pal M and Koul S: Role of p38 MAP kinase signal transduction in solid tumors. Genes Cancer 4: 342-359, 2013.

24. Che JP, Li W, Yan Y, Liu M, Wang GC, Li QY, Yang B, Yao XD and Zheng JH: Expression and clinical significance of the nin one binding protein and p38 MAPK in prostate carcinoma. Int J Clin Exp Pathol 6: 2300-2311, 2013

25. Takeda K, Matsuzawa A, Nishitoh H and Ichijo H: Roles of MAPKKK ASK1 in stress-induced cell death. Cell Struct Funct 28: 23-29, 2003.

26. Soqa M, Matsuzawa A and Ichijo H: Oxidative stress-induced diseases via the ASK1 signaling pathway. Int J Cell Biol 2012: 439587, 2012.
27. Du ZX, Yan Y, Zhang HY, Liu BQ, Gao YY, Niu XF, Guan Y, Meng X and Wang HQ: Suppression of MG132-mediated cell death by peroxiredoxin 1 through influence on ASK1 activation in human thyroid cancer cells. Endocr Relat Cancer 17: 553-560, 2010.

28. Lee YM, Park SH, Shin DI, Hwang JY, Park B, Park YJ, Lee TH, Chae HZ, Jin BK, Oh TH, et al: Oxidative modification of peroxiredoxin is associated with drug-induced apoptotic signaling in experimental models of Parkinson disease. J Biol Chem 283: 9986-9998, 2008.

29. Jarvis RM, Hughes SM and Ledgerwood EC: Peroxiredoxin 1 functions as a signal peroxidase to receive, transduce, and transmit peroxide signals in mammalian cells. Free Radic Biol Med 53: 1522-1530, 2012. 